\title{
Development of $\mu$-PIC with resistive electrodes using sputtered carbon
}

\author{
Fumiya Yamane ${ }^{1, a}$, Atsuhiko Ochi ${ }^{1}$, Yasuhiro Homma ${ }^{1}$, Satoru Yamauchi ${ }^{1}$, Noriko Nagasaka ${ }^{1}$, Hiroaki Hasegawa ${ }^{1}$, \\ Tatsuo Kawamoto ${ }^{2}$, Yosuke Kataoka ${ }^{2}$, and Tatsuya Masubuchi ${ }^{2}$ \\ ${ }^{1}$ Kobe University \\ ${ }^{2}$ Tokyo ICEPP
}

\begin{abstract}
The Micro Pixel Chamber ( $\mu$-PIC) has been developed for a hadron-collider experiment. The main purpose is detecting Minimum Ionizing Particles (MIP) under high-rate Highly Ionizing Particles (HIP) environment. In such an environment, sufficient gain to detect MIP is needed, but continuous sparks will be caused by high-rate HIP. To reduce sparks, cathodes are made of resistive material. In this report, sputtered carbon was used as a new resistive cathode. Gas gain $>10^{4}$ was achieved using an ${ }^{55} \mathrm{Fe}$ source. This value is sufficient to detect MIP without GEM or other floating structures. Also, thanks to production improvement, pixels are well aligned in the entire detection area.
\end{abstract}

\section{Introduction}

The Micro Pixel Chamber ( $\mu$-PIC) [1] [2] is a 2-D gaseous imaging detector produced with PCB/FPC technology. $\mu$ PIC has been developed for many applications, such as the Electron-Tracking Compton Camera (ETCC) [3], directional dark matter search (NEWAGE experiment) [4], neutron imaging [5], and space dosimeter (PS-TEPC) [6]. Our goal includes a particle-tracking detector in a hadroncollider experiment.

One of our development targets is the new ATLAS forward muon detector called the Muon Tagger, which is being considered for installation between the end-cap calorimeter and the JD (The Disk Shielding) near the beamline $(2.7<|\eta|<4.0)$ in the long shutdown 3 of the LHC (year 2023) [7]. There is a very small area in the ATLAS high- $\eta$ region to measure the muon track, and there is large background radiation scattered near the beamline such as fast neutron and gamma ray. Requirements for the Muon Tagger are as follows.

- Granularity of a few hundred $\mu \mathrm{m}$ for multiple track separation.

- Stable operation in high-rate radiation background $\left(>100 \mathrm{kHz} / \mathrm{cm}^{2}\right)$.

- Multiple layers installed within a thickness of $5 \mathrm{~cm}$.

Taking into account the thickness requirement, MPGDs, or silicon detectors, are considered. In case MPGDs are used, high-rate hadronic particles, mainly gas nuclei recoiled by fast neutrons, will cause continuous sparks [8]. Those particles are called Highly Ionizing Particles (HIP). Using high-resistive electrodes is the best way to reduce the sparks. In the case of $\mu$-PIC, strong spark reduction was shown by using resistive cathodes [9]. Sparks

\footnotetext{
a e-mail: fumiyamane@stu.kobe-u.ac.jp
}

are suppressed by voltage drop on the resistive electrode. If the resistivity is high, sparks are strongly reduced, but the voltage drop becomes large. If HIP are incident at too high a rate, the voltage supply cannot supply enough current, a voltage drop is generated and the electric field is reduced. Thus, resistivity should be adjusted to the radiation environment precisely. Also, with respect to the granularity, resistive electrodes should be patterned finely on the scale of a few $\mu \mathrm{m}$. For those requirements, sputtered carbon [10] is a very promising material. In this paper, we report the detector design and gas gain measurement of $\mu$-PIC with resistive cathodes using sputtered carbon.

\section{Detector concept}

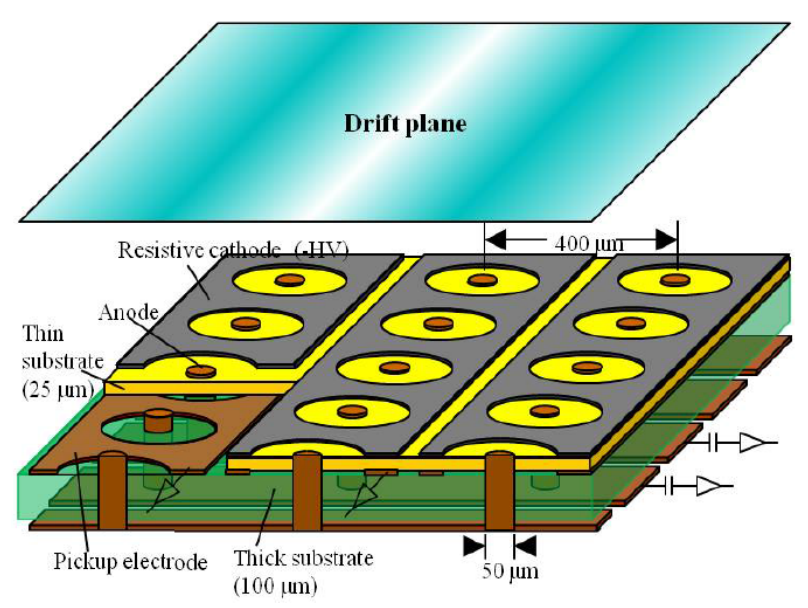

Figure 1. Schematic view of $\mu$-PIC with resistive cathodes. 
Figure 1 shows a schematic view of the $\mu$-PIC with resistive cathodes. Isolated pixels are arranged by $400 \mu \mathrm{m}$ pitch with holes $250 \mu \mathrm{m}$ in diameter on the substrate. Each pixel has an anode pin, which is connected to the readout strip through the thick substrate, and a surrounding cathode. Cathode electrodes are made of resistive material and pickup electrodes are placed under cathode electrodes. Charges are induced to pickup electrodes from resistive cathodes. Because this capacitive readout does not require any capacitors for $\mathrm{AC}$ coupling, detector construction is simplified. In our previous research [9], the $\mu$-PIC, in which cathodes were made by carbon-loaded polyimide as a resistive material, showed strong spark reduction in the fast neutron irradiation test. The spark counting rate was $10^{3 \sim 5}$ times lower than normal $\mu$-PIC with gas multiplication around $10^{4}$. This means that resistive $\mu$-PIC has high potential for both MIP detection and HIP tolerance.

More granularity and precise control of resistivity are needed for usage as a Muon Tagger; however, carbonloaded polyimide is not so uniform, a feature that makes difficult the fine patterning and the precise control of the resistivity. As mentioned, sputtered carbon is a very promising material. It has been developed for the resistive strips of ATLAS Micromegas, and its properties have been studied well [10]. Fine patterning is available $(<50 \mu \mathrm{m})$ using a liftoff process. By varying thickness (a few hundred to a few thousand $\AA$ ) and nitrogen doping, resistivity can be controlled between $50 \mathrm{k} \Omega / \mathrm{sq}$. to $4 \mathrm{G} \Omega / \mathrm{sq}$. Figure 2 shows 3-D images of a pixel of the $\mu$-PIC with resistive cathodes made of carbon-loaded polyimide and sputtered carbon, respectively.

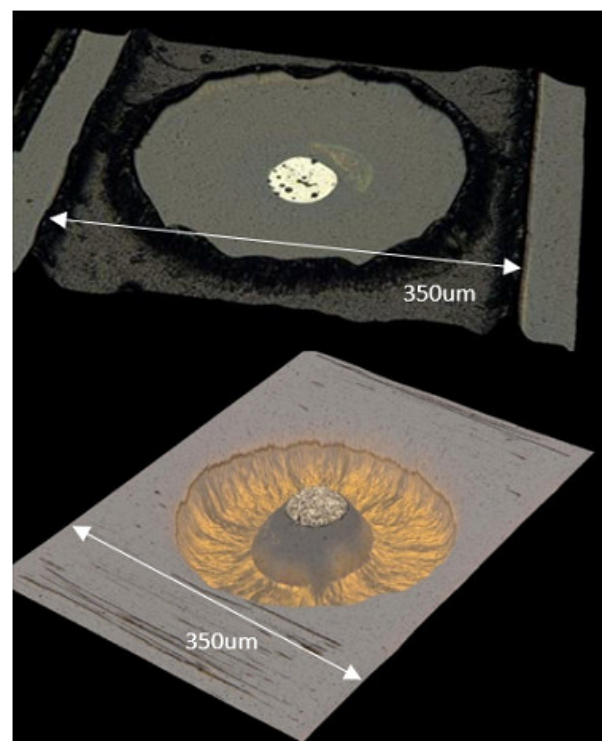

Figure 2. 3-D images of pixels of resistive $\mu$-PIC. Top: carbonloaded polyimide. Bottom: Sputtered carbon. Compared to carbon-loaded polyimide, great uniformity and flatness of sputtered carbon can be seen.

\section{Production improvement}

Manufacturing processes were done by Raytech Inc., and carbon sputtering was done by Be-Sputter Co., Ltd. Figure 3 shows our first trial. Cathode electrodes and pickups were formed by double-sided mask; then anode pins were formed by polyimide etching and nickel plating. This layer is called the top substrate. The width of cathode pickups is smaller than the resistive cathodes in order to reduce the dependence from the electrical field. Next, the thick substrate was laminated under the top substrate. This layer is called the bottom substrate. The bottom surface was covered by a copper layer for anode electrodes, which were connected to top anode pins by laser drilling and nickel plating. This process caused a misalignment of pixels because the top substrate could not be seen from the bottom side. Thus, the anode electrodes were not connected to top anode pins. To solve this problem, we used transparent dry resist for the bottom substrate. We could see the top substrate and pixels resulted aligned well by the photolithographic process. Connection to top anode pins and patterning of anode electrodes were done by copper sputtering and nickel plating. These improved processes are shown in Figure 4.

New prototypes have a $10 \times 10 \mathrm{~cm}^{2}$ detection area with 256 anode/cathode strips. Figure 5 shows a microscopic image of the detector surface. Pixels are well aligned, and carbon sputtered cathodes are properly formed in the entire detection area. A 3-D image of a pixel is also shown in Fig 2 , bottom

1. Top substrate $25 \mu \mathrm{m}$ double-sided kapton

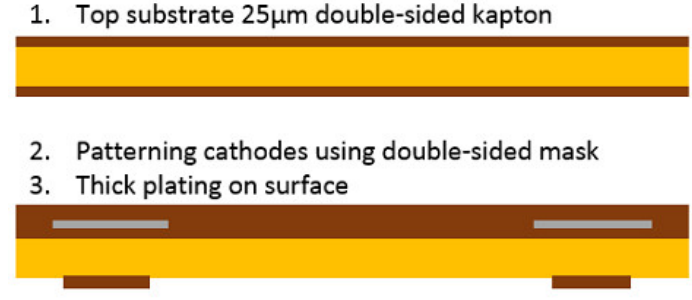

4. Etching and plating for top anode

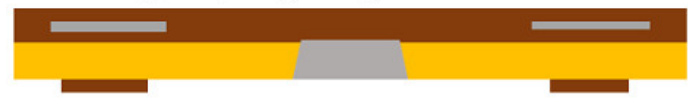

5. Laminating bottom substrate

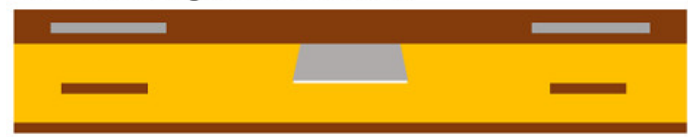

6. Laser drilling and plating for anode connection

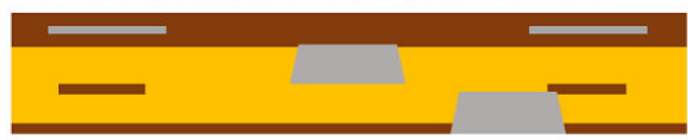

Figure 3. Previous manufacturing processes. In the laser drilling process, the top substrate cannot be seen; thus some pixels are misaligned. 


\section{Laminating $75 \mu \mathrm{m}$ dry resist as bottom substrate}

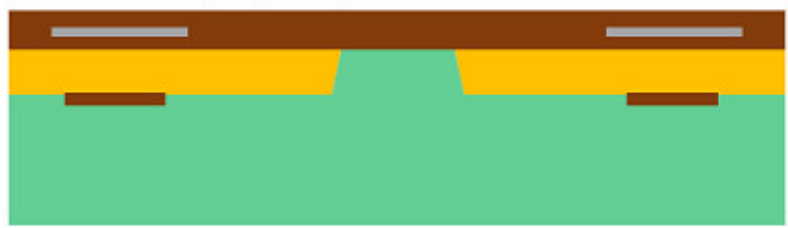

\section{Photo mask \& exposure}

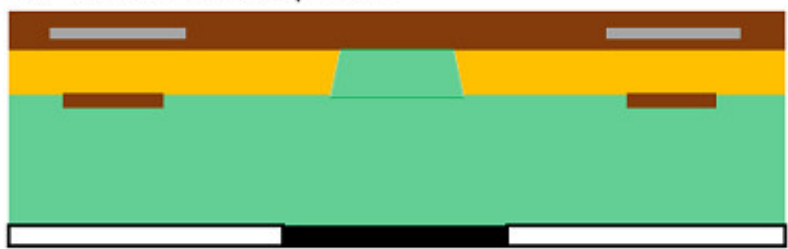

3. Developing

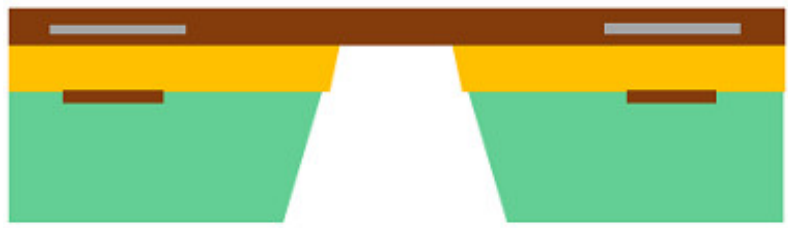

4. Copper sputtering for anode connection

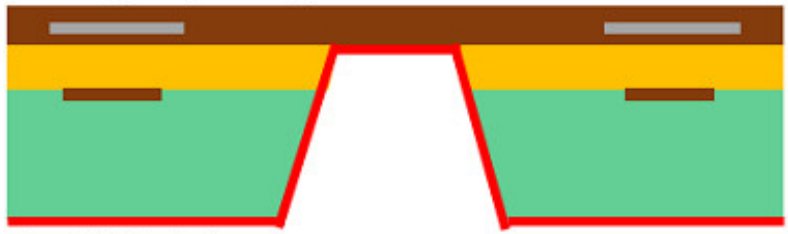

5. Nickel plating

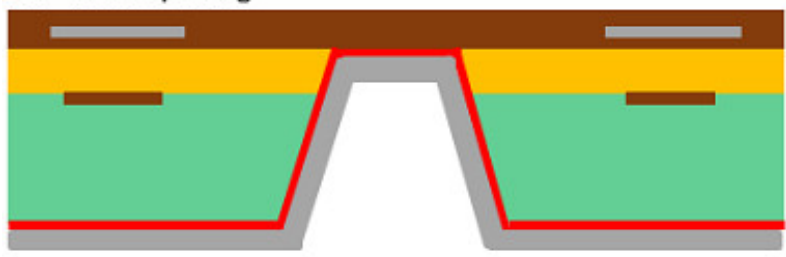

6. Carbon sputtering with liftoff

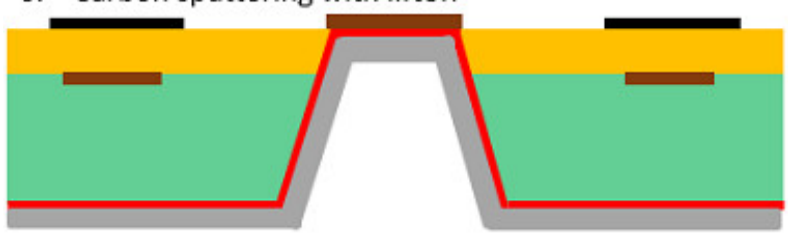

Figure 4. Improved processes. Manufacturing processes of the top substrate are the same as before. By using transparent dry resist and photolithography, pixels are well aligned in the entire detection area.

\section{Operation test}

The gas gain measurement of new prototypes was performed using an ${ }^{55} \mathrm{Fe} 5.9 \mathrm{keV} \mathrm{X}$-ray source. The gas mixture was Ar: $\mathrm{C}_{2} \mathrm{H}_{6}=90: 10$. Drift field was set to $2 \mathrm{kV} / \mathrm{cm}$ with $5 \mathrm{~mm}$ drift gap. Negative HV was supplied to the resistive cathodes and the potential of the anodes was set to 0 . Signals were read by cathode pickup electrodes, amplified by ATLAS ASD preamplifier [11], and then measured by Amptek's MCA-8000D Digital Multichannel Analyzer. Figure 6 shows the result of the measurement. The maxi-

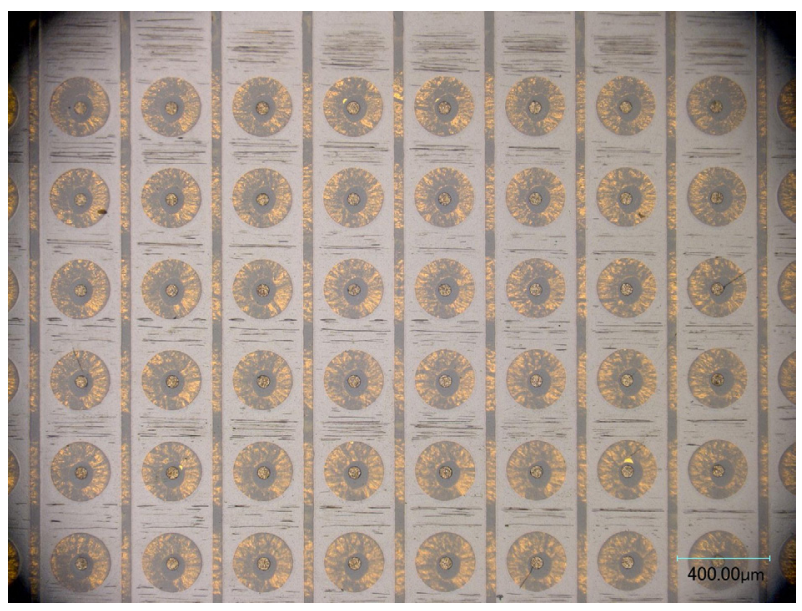

Figure 5. Microscopic image of a new prototype. The readout pitch is $400 \mu \mathrm{m}$. Pixels are well aligned, and carbon sputtered cathodes are properly formed in the entire detection area.

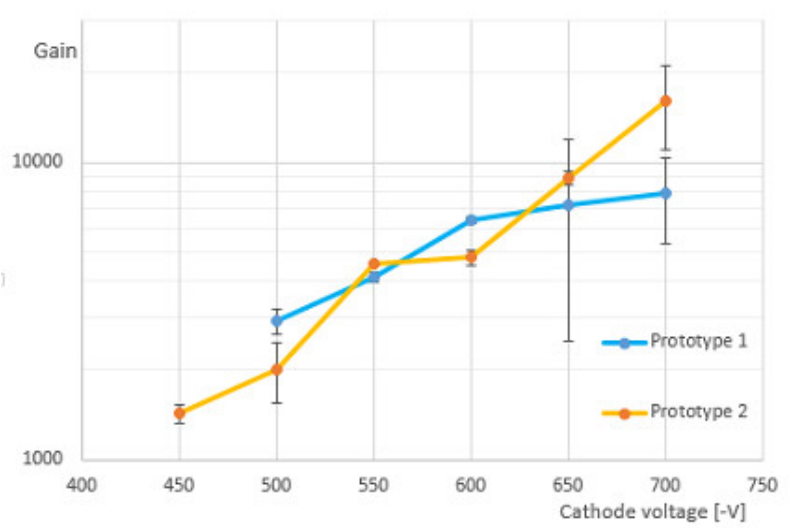

Figure 6. Gas gain of new prototypes versus cathode voltage. Anode voltage was set to 0 . The gas mixture was Ar: $\mathrm{C}_{2} \mathrm{H}_{6}=90: 10$. Drift field was set to $2 \mathrm{kV} / \mathrm{cm}$. Signals were read by cathode pickup electrodes. Gain $>10^{4}$ was obtained.

mum gain is around $10^{4}$. Although plots were finished at $\left(-700 \mathrm{~V}, \sim 10^{4}\right)$, this is not due to discharge effects but to the ASD saturation.

Although signals were obtained from the cathode pickups, they could not be obtained from the anodes. Additionally, supplying positive HV to the anodes and setting the cathodes to 0 (this operation is rather typical for $\mu$ PIC), signals could not be obtained by both anodes and cathode pickups. Gain reduction by continuous irradiation was also observed. After an irradiation for a few hours, the gas gain decreased by a factor two (Figure 7).

The observed problems are caused by anode strips not connected to the top anode because dry resist remained around it. Figure 8 shows an electron microscopic image of the cross section of the new prototypes. Plating of the anode was properly formed but did not reach the top anode. Thus, gain variation was caused by the charging-up effect on the top anodes. Recently, processes of anode con- 
nection have been improved, and new detectors have been produced.

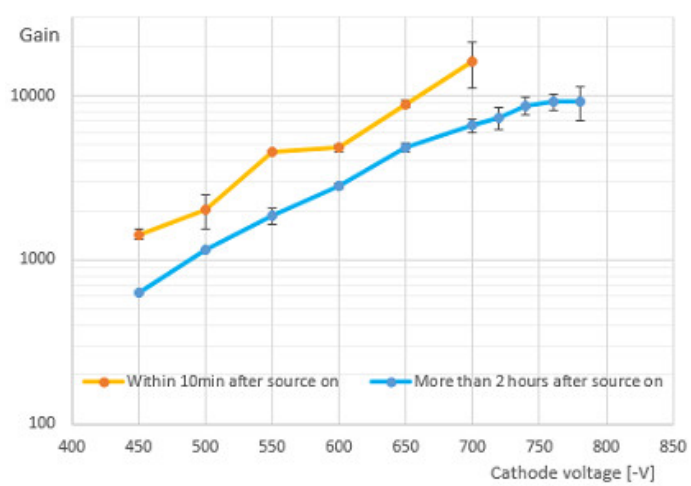

Figure 7. Gain variation due to charging-up effect on top anodes. Gain reduction occurred during several hours from source on.

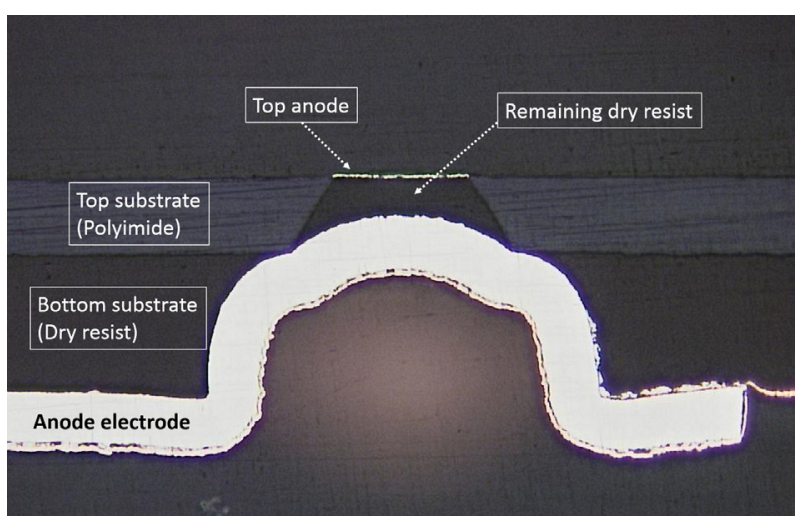

Figure 8. Electron microscopic image of cross section of a new prototype. Anode strips were not connected to top anode due to dry resist remaining around it.

\section{Conclusion}

$\mu$-PIC with resistive cathodes using sputtered carbon was developed and tested. By using dry resist as the bottom substrate and photolithography, pixels were well aligned in the entire detection area. High gas gain $\left(>10^{4}\right)$ was achieved using an ${ }^{55} \mathrm{Fe} 5.9 \mathrm{keV} \mathrm{X}$-ray source and discharge did not occur. This means that this new detector has the potential to detect MIP without preamplification by GEM or other floating structures such as foils or meshes. However, these prototypes are not perfect because the anode strips were not connected to top anodes. Processes were improved, and new prototypes were produced. We are planning to perform a fast-neutron irradiation test to check HIP tolerance in 2016.

We would like to thank RAYTECH Inc. and BeSputter Co., Ltd. This work was supported by JSPS KAKENHI Grant Numbers 26104707, 26610069.

\section{References}

[1] A. Ochi et al., NIM A 471, 264 (2001)

[2] A. Ochi et al., NIM A 478, 196 (2002)

[3] T. Tanimori, The Astrophysical Journal 810, 28 (2015)

[4] K. Nakamura, PTEP 043F01, (2015)

[5] J. D. Parker, NIM A 497, 23 (2013)

[6] Y. Kishimoto, NIM A 732, 591, (2013)

[7] The ATLAS Collaboration, ATLAS Phase-II Upgrade Scoping Document (CERN-LHCC, 2015)

[8] H. Raether, Electron avalanches and breakdown in gases (Butterworth, London U.K., 1964)

[9] A. Ochi et al., JINST 9 C01039, 1 (2014)

[10] A. Ochi et al., PoS TIPP2014, 351 (2014)

[11] ATLAS TGC collaboration, Amplifier-ShaperDiscriminator ICs and ASD Boards (Production Readiness Review Report, 1999) 